\title{
Application of Nomogram Based on Radiomics Label in Prediction of Treatment Scheme for Lumbar Disc Herniation
}

\section{Gang Yu}

Graduate School of Jiangxi University of Traditional Chinese Medicine

\section{Wenlong Yang}

Affiliated Hospital of Jiangxi University of Chinese Medicine

Jingkun Zhang

Affiliated Hospital of Jiangxi University of Chinese Medicine

Qi Zhang

Affiliated Hospital of Jiangxi University of Chinese Medicine

\section{Jian Zhou}

Affiliated Hospital of Jiangxi University of Chinese Medicine

\section{Yuan Hong}

Affiliated Hospital of Jiangxi University of Chinese Medicine

Jiaojiao Luo

Graduate School of Jiangxi University of Traditional Chinese Medicine

Kangyu Zhang

Jiangxi University of Traditional Chinese Medicine

\section{Zhidan Yang}

Jiangxi University of Traditional Chinese Medicine

Hong Tu (D449137781@qq.com )

Affiliated Hospital of Jiangxi University of Chinese Medicine

\section{Research Article}

Keywords: Radiomics, Nomogram, Lumbar Disc Herniation, Artificial Intelligence, Prediction Model, Surgical Treatment, Conservative Treatment

Posted Date: April 28th, 2021

DOI: https://doi.org/10.21203/rs.3.rs-440032/v1

License: (c) (1) This work is licensed under a Creative Commons Attribution 4.0 International License. Read Full License 


\section{Abstract}

Objective: To investigate and verify the efficiency and effectiveness the the value of nomogram based on radiomics label in predicting the treatment of lumbar disc herniation (LDH).

Methods: The clinical medical records and imaging data of 200 patients with LDH diagnosed in the Affiliated Hospital of Jiangxi University of Traditional Chinese Medicine in the past 3 years have been analyzed retrospectively. The collected cases are randomly divided into a training group $(n=140)$ and a test group $(n=60)$ with ratio of 7:3. Two radiologists with experience in reading orthopaedics images are independently segmented ROI, the whole intervertebral disc with the most obvious protrusion in the sagittal plane $\mathrm{T}_{2} \mathrm{WI}$ of lumbar MRI as a mask (ROI) are sketched. The LASSO algorithm is used to filter the features after extracting the radiomics features. The multivariate Logistic regression model is used to construct a quantitative imaging Rad-Score for the selected features with non-zero coefficients. The radiomics labels and nomogram were evaluated using the receiver operating characteristic curve (ROC) and the area under the curve (AUC). The calibration curve is used to evaluate the consistency between the nomogram prediction and the actual treatment plan. The DCA decision curve is used to evaluate the clinical applicability of nomogram.

Result: Following the feature extraction, 11 radiomics features are used to construct the radiomics label for predicting the treatment plan of $\mathrm{LDH}$, The nomogram was then constructed. The AUC was 0.930 (95\% Cl:0.865-0.995) with a sensitivity of $96.8 \%$ and a specificity of $90 \%$. The calibration curve shows that there is a good consistency between the prediction and the actual observation. The DCA decision curve analysis shows that the nomogram of imaging group has a great potential for clinical application when the risk threshold is over $10 \%$.

Conclusion: The nomogram based on radiomics label has a good predictive value for the treatment of $\mathrm{LDH}$ and therefore can be used as a reference for clinical decision-making.

\section{Introduction}

In the recent years, the irregular life and rest of modern people lead to the incidence of Lumbar Disc Herniation (LDH) continues to rise. Even after treatments, the disease still recurs frequently. Meanwhile, the onset age becomes younger and more serious affecting daily life ${ }^{[1]}$. The lumbar disc herniation of lumbar degenerative changes referring to a variety of reasons, including external force damage, and longterm lumbar caused by bad habits. such as part of some or all of the rupture, and highlight the nucleus pulposus, rupture, stimulate or oppressed nerve root, horsetail nerve palsy is a clinical syndrome, is one of a common cause of low back and leg pain. Surgeries and conservative treatments are effective methods for the treatment of lumbar disc herniation. Surgical treatment can be mainly divided into traditional surgery and minimally invasive surgery. Through data analysis, some researchers believe that surgery has obvious advantages compared to conservative treatments in improving lumbar and leg pain, muscle paralysis, low quality of life and adverse reactions caused by this disease ${ }^{[2]}$. However, it doesn't mean 
that surgery is the first choice for all patients with a lumbar disc herniation, most patients with a lumbar disc herniation can be treated conservatively, Studies have confirmed that conservative treatments can effectively alleviate the symptoms of LDH, and thus it is considered as the first-line choice for most patients. The early efficacy of LDH is no worse than that of surgery by improving the lifestyle, physical therapy, traditional Chinese medicine treatment and routine use of drugs ${ }^{[3]}$. From the most basic bed rests, tractions, functional exercises, to the traditional internal and external use of Chinese medicine, acupuncture and massage and palasy, to combine traditional Chinese and western medicine of traditional Chinese medicine preparation sacral canal injection, small needle knife therapy, as well as the comprehensive treatment, there are many types and the conservative treatment of LDH curative effect is distinct, and with the progress of medicine is growing and changing ${ }^{[4]}$.In the selection of surgical treatment, doctors should follow the surgical indications of LDH:i) LDH is clearly diagnosed and ineffective after 3 months of conservative treatment, affecting daily life and work; ii)The pain is so intense that makes patients difficult to move or even sleep. The CT or MRI showed rupture of the annulus fibrosus and dissociation of the nucleus pulposus; iii)There was muscle paralysis and cauda equina nerve damage ${ }^{[5]}$. It can be seen that surgical indications are highly subjective. It indicates thathow to choose the most appropriate treatment plan has been a problem of perplexing clinicians. Improper selection is likely to result in an inadequate or excessive treatment. At present, there is no quantitative

method to judge the treatment plan ${ }^{[6]}$. Radiomics ${ }^{[7]}$ is a method that can convert digital medical images into high-dimensional data that can be mined. It can convert visual image information into deep features for quantitative research and reveal the information contained in the images that reflect the underlying pathophysiology. The purpose of this study is to predict surgical or conservative treatments for lumbar disc herniation using a nomogram that is based on radiomics label.

\section{Data And Methods}

\section{Clinical data}

Part of the clinical medical records and imaging data of 200 patients with lumbar disc herniation diagnosed in the Affiliated Hospital of Jiangxi University of Traditional Chinese Medicine in the past 3 years are retrospectively collected. They are randomly divided into a training group and a validation group in a ratio of 7:3. The inclusion criteria are defined as follows: (1) The patient had been clearly diagnosed as lumbar disc herniation without limitation of personal basic information; (2) Lumbar MRI was performed in our hospital, and the imaging information at least included OSag- $\mathrm{T}_{2} \mathrm{WI}$ sequence and OAx$\mathrm{T}_{2} \mathrm{WI}$ sequence. The case exclusion criteria are defined as follows: (1) The image quality is poor, and it is difficult to outline the region of interest (ROI) or to extract the image omics features; (2) Patients with other lumbar diseases; (3) Complicated with malignant tumor; (4) People with schizophrenia or severe 
mental disorders; (5) Severe osteoporosis; (6) vertebral body compression; (7) Previous diagnosis and treatment of LDH.

\section{MRI examination method}

The magnetic resonance imaging scanners named Signa Hde 1.5T and Discovery MR750冈3T区 from General Electric Company(GE) is used. After lunching the exam to be ready for the diagnosis,, the spinal phasing front coil is used and the patient wearing a hearing protection is instructed not to move during the examination. The lumbar scan protocol is invoked during the scanning. The scanning sequence and azimuth are included FSE-T1WI sequence in the sagittal plane, FSE- ${ }_{2}$ WI sequence is in the sagittal plane, FSE- $T_{2} \mathrm{WI}$ sequence is in the sagittal plane, and FSE-T 2 WI sequence is in the transverse plane. Due to the possibility of disc-bone overlap in cross-sectional $\mathrm{T}_{2} \mathrm{WI}$ sequences, which affects image representativeness, only the sagittal $\mathrm{T}_{2} \mathrm{WI}$ sequences were used in this study. The main parameters includes TR $2000 \mathrm{~ms}$, TE $120 \mathrm{~ms}$, layer thickness $4 \mathrm{~mm}$, layer spacing $0.5 \mathrm{~mm}$, matrix $320 \star 224$, average acquisition times twice, and field of view $320 \mathrm{~mm} * 320 \mathrm{~mm}$.

\section{Image segmentation and feature extraction}

3.1 Segmentation of ROI: Two radiologists who have many years of experience in reading orthopaedics films independently use the ITK-SNAP\v3.6.0, http://www.itksnap.org/\software ${ }^{[8]}$, respectively marking the most prominent intervertebral discs in the sagittal $\mathrm{T}_{2} \mathrm{WI}$ sequence of lumbar $\mathrm{MRI}$, and synthesizing the region of interest $(\mathrm{ROI})$.

3.2 Feature extraction: The Pyradiomics package (v3.0.1, http://pyradiomics.

readthedocs.io/) of the open source Python (v3.8.5) software is used to complete the feature extraction and screening of radiomics. The image preprocessing includes resampling into $3 \times 3 \times 3$ isotropic voxels, extracting the original image, Log and wavelet features, and normalizing and discretizing at the same time (BinWidth :5). The obtained data are analyzed. First, the consistency test between observers is carried out to calculate the interclass correlation coefficient (ICC) between the features extracted from the ROI drawn by two radiologists, so as to evaluate the repeatability of the radiomics features drawn by two radiologists. When ICC value is larger than 0.75 , consistency of feature extraction is good ${ }^{[9]}$.

3.3 Establishment and evaluation of radiomics labels: Using the Least absolute shrinkage and selection operator (LASSO) for feature selecting. The parameter lambda $(\lambda)$ of Lasso regression model is selected by cross-validation, and the $\lambda$ with the smallest model error was selected to retain the feature when the coefficient was not equal to zero. The multivariate logistic regression model is used to construct quantitative radiomics labels for the selected features with non-zero coefficients. A weighted linear combination of the coefficients of non-zero coefficient features is used to obtain the radiomics label score (Rad-Score) for each patient. The receiver operating characteristic (ROC) curve ${ }^{[10]}$ is used to 
evaluate the efficacy of the radiomics label in predicting the treatment plan of lumbar disc herniation. The area under the curve $(A \cup C)^{[11]}$, sensitivity, specificity and accuracy is calculated.

3.4 Establishment and evaluation of nomogram based on radiomics label: The R statistical software package is used to establish and to evaluate the consistency between the prediction of the radiomics nomogram and the actual choice of treatment plan The Hosmer-Lemeshow test is used to analyze the degree of fit of the nomogram ${ }^{[12]}$. The Harrell consistency index (C-index) is measured to quantify the discriminative power of radiomics ${ }^{[13]}$. To evaluate the clinical application of the nomogram, we have used the decision curve analysis (DCA) to calculate the net benefit under different threshold probabilities. The net benefit is defined as that the proportion of true positives minus the proportion of false positives plus the relative harm of false and false negative results ${ }^{[14]}$.

\section{Statistical analysis}

The R statistical software $\Downarrow$ v4.0.3邓http://www.rproject.org/囚is used for statistical analysis. Counting data are expressed as frequency. The Chi-square test or Fisher's exact probability method is used for comparison between the training and testing groups. The $\mathrm{W}$ test is used to verify whether the measurement data follow a normal distribution. If not, $x \pm s$ is used to represent the measurement data. The independent sample $t$ test is used for comparison between the two groups. If the data does not follow a normal distribution with the median (upper and lower quartile), the comparison between the two groups using Mann-Whitneyu test.Lasso and logistic regression is modeled by the "glmnet" package. The software package "rms" and "regplot" are used to construct the nomogram and establish the correction curve of the nomogram. The ROC curve is drawn by the package "pROC"; the C-index is calculated by the package "Hmisc"; the Hosmer-Lemeshow test is carried out in the package "ResourceSelection"; The DCA decision curve is drawn within the package "rmda". The $\mathrm{P}<0.05$ is considered as a statistical significance.

\section{Result}

\section{Case data}

A total of 200 patients diagnosed with "lumbar disc herniation" are included, and all of them visited the Affiliated Hospital of Jiangxi University of Traditional Chinese Medicine in the past three years. Among them, 100 patients were treated by surgical method and 100 patients were treated by conservative method. Among them, there were 98 males and 102 females, aged between 21.0 89.0 (55.2 15.7$)$ years. The collected cases are randomly divided into a training group $(n=140)$ and a validation group $(n=60)$ in a 7:3 ratio following the random sampling approach.

\section{Establishment and evaluation of radiomics labels}

A total of 1083 features have been extracted using the $T_{2} \mathrm{Wl}$ of the patient's lumbar sagittal plane (see Figure 1), among which 313 features with ROI greater than 0.75 were delineated by two physicians. After removing highly correlated features, 139 features are remained. The Lasso regression is performed for 
feature screening and 11 radiomics features ar obtained. Among the radiomics, there are 5 first-order features, including Mean, Interquartile Range, Maximum, Root Mean Squared (RMS) and Entropy; there are 4 features of gray level co-occurrence matrix, including Correlation, Inverse Variance, Joint Energy, and Informational Measure of Correlation (IMC1); The gray level correlation matrix feature is the Dependence Non-Uniformity Normalized $\triangle \mathrm{DNN}$ ); One matrix feature of Gray size area, which is Gray Level Variance (GLV). Based on the 11 selected radiomics characteristics and their coefficients, a radiomics label is constructed to predict the treatment plan of lumbar disc herniation. The best $\lambda$ value obtained through calculation is 0.030 as presented in Figure 2 and Figure 3.. A linear formulation is used to calculate the Rad-score for each patient to predict the treatment plan for lumbar disc herniation. The formula is as follows:

Rad-Score $=18.298+[0.002 \times$ diagnostics_Image-original_Mean]-[0.331×log-sigma-4-0-mm3D_glcm_Correlation]+[4.513×log-sigma-5-0-mm-3D_glcm_InverseVariance]-

[22.778×original_glcm_JointEnergy]+[3.133×wavelet-HHH_gldm_DependenceNonUniformityNormalized]+ [0.009 $\times$ wavelet-HHL_firstorder_InterquartileRange]-[0.002×wavelet-HLL_firstorder_Maximum][0.085×wavelet-HLL_firstorder_RootMeanSquared]-[0.0008×wavelet-HLL_glszm_GrayLevelVariance]+ [1.855×wavelet-LLH_firstorder_Entropy]+[1.509×wavelet-LLL_glcm_Imc1].

In the normal distribution test of Rad-score, the $P$ values of both the training group ( $W=0.99405, p-$ value $=0.8323)$ and the validation group $(W=0.98771$, $p$-value $=0.8079)$ are greater than 0.05 , indicating that the Rad-score follows a distribution. The Rad-Score in the training group is $0.336 \pm 0.656$ and $-0.336 \pm 0.577$ in the surgery and conservative patients, respectively, and the difference is statistically significant $\left(t=-6.4247, p\right.$-value $\left.=2.103 \times 10^{-09}<0.05\right)$. In the validation group, the values were $0.523 \pm 0.546$ and $-0.531 \pm 0.614$ in the patents treated by the surgical and conservative measures, respectively. The difference is statistically significant $\left(t=-7.0145\right.$, $p$-value $\left.=3.252 \times 10^{-09}<0.05\right)$. The Rad-Scores of each patient in the training group and the validation group are shown in Figure 4 and Figure 5, respectively. In the training group, the ROC curve AUC of radiomics label for predicting the treatment of lumbar disc herniation is $0.766(95 \% \mathrm{Cl}: 0.689-0.843)$, with a sensitivity of $59.4 \%$, a specificity of $78.9 \%$, and an accuracy of $67.1 \%$ (as shown in Figure 6). In the validation group, the AUC is 0.895 (95\%Cl: $0.817-0.974$ ), the sensitivity is $82.8 \%$, the specificity is $87.1 \%$, and the accuracy is $83.3 \%$ ( see Figure 7 ).

\section{Construction and validation of radiomics nomogram}

A nomogram is constructed based on the radiomics label (Figure 8 ). The $\mathrm{C}$-index of the radiomics nomogram delivers the treatment plan that has an AUC value of 0.930 (95\% Cl:0.865-0.995), sensitivity of $96.8 \%$, specificity of $86.2 \%$, and accuracy of $90 \%$ (as presented Figure 9 ) for lumbar disc herniation The correction curves show a good agreement between the training group (Figure 10) and the validation group (Figure 11) between the prediction and the selection of the actual treatment regimen in the clinic. Hosmer-Lemeshow test showd that the difference is not statistically significant $(X$-squared $=12.171$, $p$ value $=0.1437>0.05$ ), indicating no deviation from fitting. DCA decision curve analysis (Figure 12) indicates that when the risk threshold is greater than $10 \%$, the use of radiomics label nomogram to 
predict the treatment of lumbar disc herniation has increased the net benefit more than that when using all surgical treatment and all conservative treatment.

In Figure 10 and Figure 11, the blue curve represents the ideal prediction performance the red line represents the actual prediction performance, and the green line represents the corrected prediction performance. The prediction accuracy is higher when the distance between the lines is smaller. As can be seen from the figures, there is a good agreement between the treatment plans predicted by the nomogram and the actual clinician's choice of treatment for lumbar disc herniation.

As can be seen from the figure, when the risk threshold is greater than $10 \%$, the radiomics labeling nomogram approach is superior to treating all patients as surgical or conservative.

\section{Discussion}

In this study, we have developed and validated a nomogram that consists of a radiomics label (Radscore) of lumbar MRI for predicting treatment of lumbar disc herniation, which have shown a good predictive performance as confirmed by the prediction plan with an AUC of 0.930 (95\% Cl:0.865-0.995), a sensitivity of $96.8 \%$, a specificity of $86.2 \%$, and an accuracy of $90 \%$.Our results suggest that the radiomics label nomogram based on lumbar MRI can be used as a quantitative predictive tool to provide clinicians with reference for treatment choice.

In the past, researchers have tried to combine multidimensional biomarkers with the great potential clinical value identified through high-throughput techniques to model diseases for obtaining satisfactory results. The biomarkers included in the model cover different biological scales from molecular to phenotypic ${ }^{[15]}$. Radiomics in the application of skeletal muscle system is usually in terms of bone tumors, such as bone disease diagnosis and differential diagnosis of tumor ${ }^{[16,17]}$, prediction ${ }^{[18]}$ of tumor complications, the prognosis of tumor treatment pathologic grading ${ }^{[19-20]}$ and tumor, a small study applies beside the osteoporosis ${ }^{[21]}$, vertebral abscess ${ }^{[22]}$, temporo-mandibular joint osteoarthritis [23], postoperative infection and inflammation ${ }^{[24]}$, and so on. Few radiomics studies have been conducted on LHD. The Schulthess Klinik orthopaedic team in Zurich, Switzerland, has developed a clinical prognostic model tool ${ }^{[25]}$ that can predict the surgical outcome of disc herniation. The model can help doctors to provide patients with truthful information and reasonable expectations for the surgical outcome. In China, there are few similar studiesthat are basically the construction of diagnostic models related to lumbar disc herniation. For example, Li Xiangrong ${ }^{[26]}$ established the surface EMG recognition model of the compressed nerve root of lumbar disc herniation through three different pattern recognition methods, and the results showed that a stable diagnostic efficiency was achieved..

In this study, 11 radiomics features are screened out, which aree the first-order gray nomogram feature ( $\mathrm{n}$ $=5)$, the gray level co-occurrence matrix $\operatorname{GLCM}(n=4)$, the gray level correlation matrix $\operatorname{GLDM}(n=1)$, and the gray size region matrix GLSZM $(n=1)$. Except for the 5 features of the first-order gray nomogram, the other 6 features belong to higher order features of the spatial distribution of the pixel points, which 
indicates that the first-order 2D and 3D features visible to the naked eye are not enough for the image description of lumbar disc herniation, and they need to be combined with the high-dimensional features that cannot be recognized by the naked eyes. Therefore, the 11 quantitative radiomics features included in this study can reflect deeper information of the images of lumbar disc herniation from different perspectives.

In this study, we first investigated the role of an MRI-based radiomics label (Rad-score) in predicting treatment options for lumbar disc herniation. The results indicate that in the training group, the ROC curve AUC of radiomics label for predicting the treatment of lumbar disc herniation was 0.766 (95\% Cl:0.6890.843), the sensitivity, specificity and accuracy are $59.4 \%, 78.9 \%$ and $67.1 \%$, respectively; In the validation group, the AUC is 0.895 (95\% Cl:0.817-0.974), the sensitivity is $82.8 \%$, the specificity is $87.1 \%$, and the accuracy is $83.3 \%$. The score magnitude positively relates to the inclination of surgical treatment.. For instance, a score greater than 0 represents the surgical treatment, and a score less than 0 represents a conservative treatment. We constructed the nomogram then through the radiomics label, with an AUC of 0.930 (95\% Cl:0.865-0.995), a sensitivity of $96.8 \%$, a specificity of $86.2 \%$, and an accuracy of $90 \%$, showing a good discriminant efficiency. At the same time, we corrected the nomogram and found that there is a good agreement between the treatment plan predicted by the nomogram and the actual clinician's choice of the treatment plan for lumbar disc herniation. The main clinical application of the nomogram is to explain the net benefit of the individual. However, discrimination efficiency and calibration may not achieve a specific level of discrimination or the clinical consequences of the degree of miscalibration ${ }^{[27,28]}$. To address this problem, clinical decision curve analysis (DCA) is used to evaluate the clinical application of the nomogram. This new strategy provides a net benefit from an in-

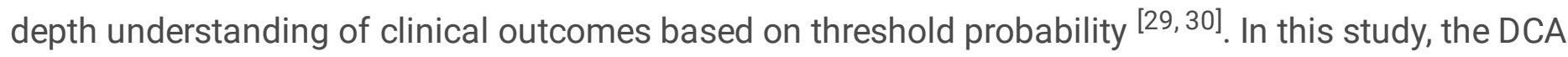
indicated that when the risk threshold was greater than $10 \%$, the radiomics labeling nomogram approach was superior to treating all patients as either surgical or conservative.

There are some limitations in this study. First, this was a retrospective study conducted in a single center with a relatively small sample size. Therefore, a multi-center validation is needed to obtain strong evidence for its clinical application. Secondly, only one sequence of sagittal $T_{2} W I$ is used in this study to extract radiomics features, and current studies have shown that multi-parameter MRI sequences can provide more information about lesions ${ }^{[31]}$. Thirdly, considering that patients may be involved in a single or multiple lesions, including clinical factors of patients that may lead to selection bias, only radiomics features are selected for the construction of the nomogram model, since the object of this study is the lumbar lesions.

\section{Conclusion}

The Nomogram based on set of radiomics labels for the treatment of lumbar disc prolapse with appropriate predictive values is capable of providing a reliable support to clinical decision-making process and to help clinicians choose for surgery and conservative treatment. In addition, the Nomogram 
has the capability to provide conservative treatment efficacy in quantitatively giving reference for clinicians to avoid inadequate and excessive treatments.

\section{Declarations}

\section{Ethics approval and consent to participate}

This retrospective study was approved by the institutional review board at Affiliated Hospital of Jiangxi University of Traditional Chinese Medicine, and the requirement of patients' informed consent was waived. All methods were carried out in accordance with relevant guidelines and regulations.

\section{Consent for publication}

Not applicable.

\section{Availability of data and materials}

The datasets used and/or analysed during the current study are available from the corresponding author on reasonable request.

\section{Competing interests}

The authors declare that they have no competing interests.

\section{Funding}

Funding comes from Science and Technology Planning Project of Jiangxi Province [Department of Science and Technology of Jiangxi Province, 20192BBG70047].

\section{Authors' contributions}

Gang Yu and Kangyu Zhang mainly wrote the first draft. Wenlong Yang corrected the manuscript. Jingkun Zhang, Qi Zhang, Jian Zhou completed the image processing part of the manuscript. Yuan Hong, Jiaojiao Luo made the pictures in the manuscript, and Zhidan Yang polished the grammar in the manuscript. Hong Tu guided the overall conduct of this research. All authors read and approved the final manuscript.

\section{Acknowledgements}

Not applicable.

\section{Authors' information}

About the author: Yu Gang (1996- ), male, from Shangrao, Jiangxi, master of medicine, resident physician, mainly engaged in the research of orthopedics and traumatology of Chinese medicine. 
Corresponding author: Tuhong (1970-), male, chief physician, master tutor, study of mainly engaged in orthopedic diseases. Email: 449137781@qq.com.

\section{References}

[1] PARKER S L, MENDENHALL S K, GODIL S S, et al. Incidence of Low Back Pain After Lumbar Discectomy for Herniated Disc and Its Effect on Patient-reported Outcomes[J]. Clin Orthop Relat Res, 2015,473(6): 1988-1999.

[2] CHEN B L, GUO J B, ZHANG H W, et al. Surgical versus non-operative treatment for lumbar disc herniation: a systematic review and meta-analysis[J]. Clin Rehabil, 2018,32(2): 146-160.

[3] ZHANG B, XU H, WANG J, et al. A narrative review of non-operative treatment, especially traditional Chinese medicine therapy, for lumbar intervertebral disc herniation[J]. Biosci Trends, 2017,11(4): 406417.

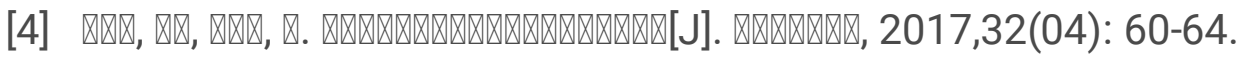

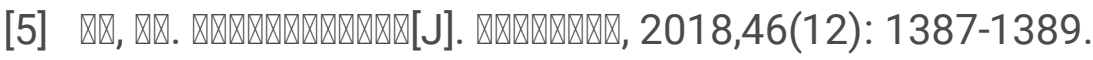

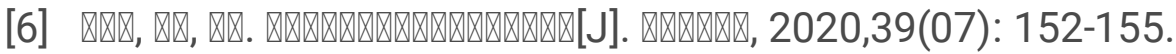

[7] GILLIES R J, KINAHAN P E, HRICAK H. Radiomics: Images Are More than Pictures, They Are Data[J]. Radiology, 2016,278(2): 563-577.

[8] YUSHKEVICH P A, PIVEN J, HAZLETT H C, et al. User-guided 3D active contour segmentation of anatomical structures: significantly improved efficiency and reliability[J]. Neuroimage, 2006,31(3): 11161128.

[9] HUANG Y Q, LIANG C H, HE L, et al. Development and Validation of a Radiomics Nomogram for Preoperative Prediction of Lymph Node Metastasis in Colorectal Cancer[J]. J Clin Oncol, 2016,34(18): 2157-2164.

[10] HOO Z H, CANDLISH J, TEARE D. What is an ROC curve?[J]. Emerg Med J, 2017,34(6): 357-359.

[11] KAMARUDIN A N, COX T, KOLAMUNNAGE-DONA R. Time-dependent ROC curve analysis in medical research: current methods and applications[J]. BMC Med Res Methodol, 2017,17(1): 53.

[12] KRAMER A A, ZIMMERMAN J E. Assessing the calibration of mortality benchmarks in critical care: The Hosmer-Lemeshow test revisited[J]. Crit Care Med, 2007,35(9): 2052-2056.

[13] WOLBERS M, KOLLER M T, WITTEMAN J C, et al. Prognostic models with competing risks: methods and application to coronary risk prediction[J]. Epidemiology, 2009,20(4): 555-561. 
[14] VICKERS A J, ELKIN E B. Decision curve analysis: a novel method for evaluating prediction models[J]. Med Decis Making, 2006,26(6): 565-574.

[15] YOUNESI E, HOFMANN-APITIUS M. From integrative disease modeling to predictive, preventive, personalized and participatory (P4) medicine[J]. EPMA J, 2013,4(1): 23.

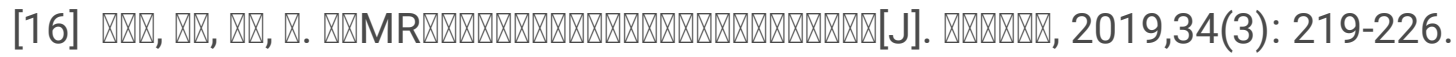

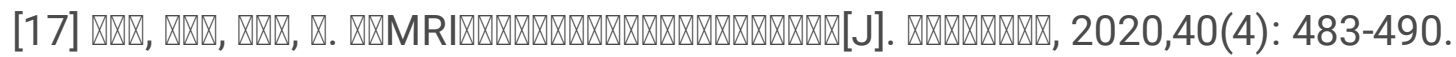

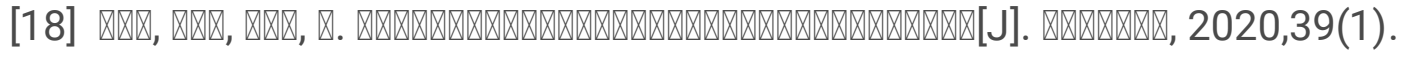

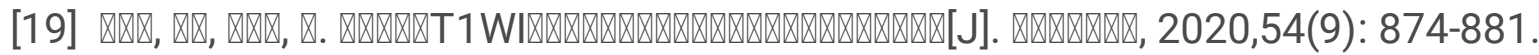

[20] WANG H, CHEN H, DUAN S, et al. Radiomics and Machine Learning With Multiparametric Preoperative MRI May Accurately Predict the Histopathological Grades of Soft Tissue Sarcomas[J]. Journal of Magnetic Resonance Imaging, 2020,51(3): 791-797.

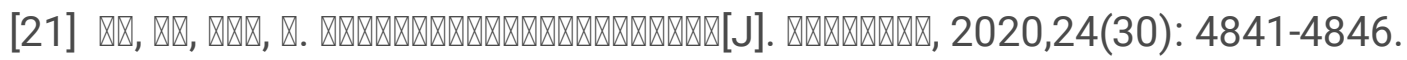

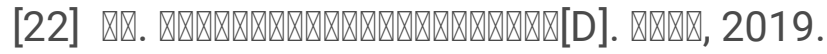

[23] BIANCHI J, de OLIVEIRA RUELLAS A C, GONÇALVES J R, et al. Osteoarthritis of the Temporomandibular Joint can be diagnosed earlier using biomarkers and machine learning[J]. Scientific reports, 2020,10(1): 8012.

[24] D'AMICO N, GANDOLFO P, VALBUSA G, et al. A radiomic approach for successful distinction of infection versus infammation in patients treated with reparative orthopaedic surgery: A pilot study[J]. European Journal of Nuclear Medicine and Molecular Imaging, 2018,45: S67.

[25] STAUB L P, AGHAYEV E, SKRIVANKOVA V, et al. Development and temporal validation of a prognostic model for 1-year clinical outcome after decompression surgery for lumbar disc herniation[J]. Eur Spine J, 2020,29(7): 1742-1751.

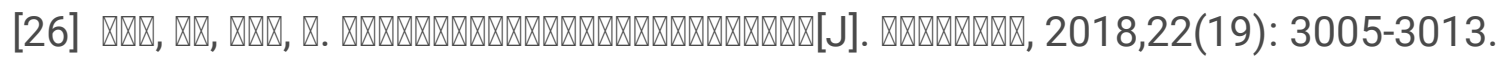

[27] LOCALIO A R, GOODMAN S. Beyond the usual prediction accuracy metrics: reporting results for clinical decision making[J]. Ann Intern Med, 2012,157(4): 294-295.

[28] Van CALSTER B, VICKERS A J. Calibration of risk prediction models: impact on decision-analytic performance[J]. Med Decis Making, 2015,35(2): 162-169.

[29] WU S, ZHENG J, LI Y, et al. A Radiomics Nomogram for the Preoperative Prediction of Lymph Node Metastasis in Bladder Cancer[J]. Clin Cancer Res, 2017,23(22): 6904-6911. 
[30] ZHANG L, DONG D, LI H, et al. Development and validation of a magnetic resonance imaging-based model for the prediction of distant metastasis before initial treatment of nasopharyngeal carcinoma: $A$ retrospective cohort study[J]. EBioMedicine, 2019,40: 327-335.

[31] LIU Z, LI Z, QU J, et al. Radiomics of Multiparametric MRI for Pretreatment Prediction of Pathologic Complete Response to Neoadjuvant Chemotherapy in Breast Cancer: A Multicenter Study[J]. Clin Cancer Res, 2019,25(12): 3538-3547.

\section{Figures}

\section{(1) Draw ROI}

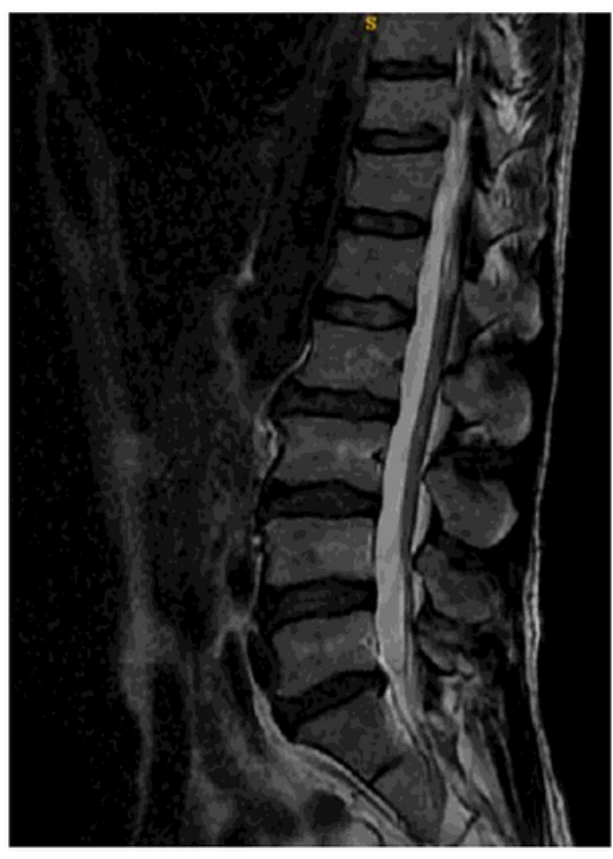

(2) Feature extraction

1083 features were

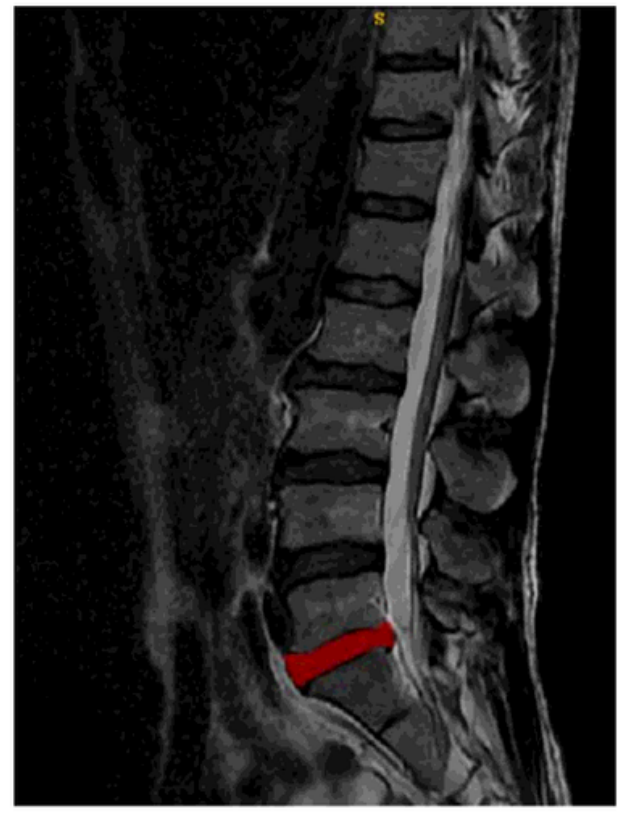

(3) Feature selection

1. Extract ICC $>0.75$ Features $(n=313)$.

2. Delete highly relevant features $(n=139)$.

3. LASSO regression selecting $(n=11)$. 
Figure 1

Radiomics feature extraction and screening process.

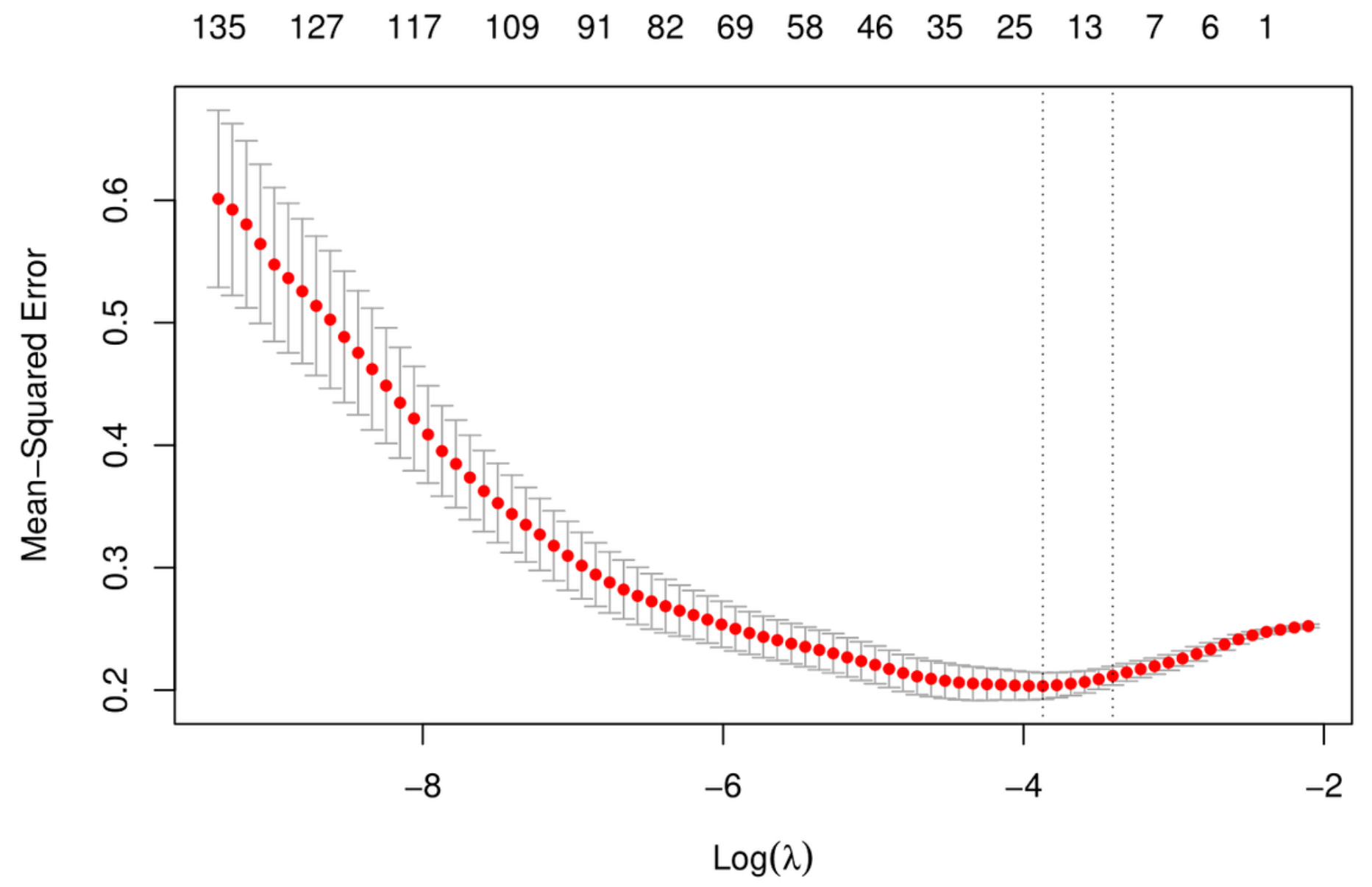

\section{Figure 2}

Feature selection in Least absolute shrinkage and selection operator (LASSO) Model.The vertical axis is the binomial deviation and the horizontal axis is the $\log (\lambda)$ value.The top number represents the number of features screened out.The smallest binomial deviation $\lambda$ is the optimal value (vertical dashed line), and the optimal $\lambda$ is 0.033 . 


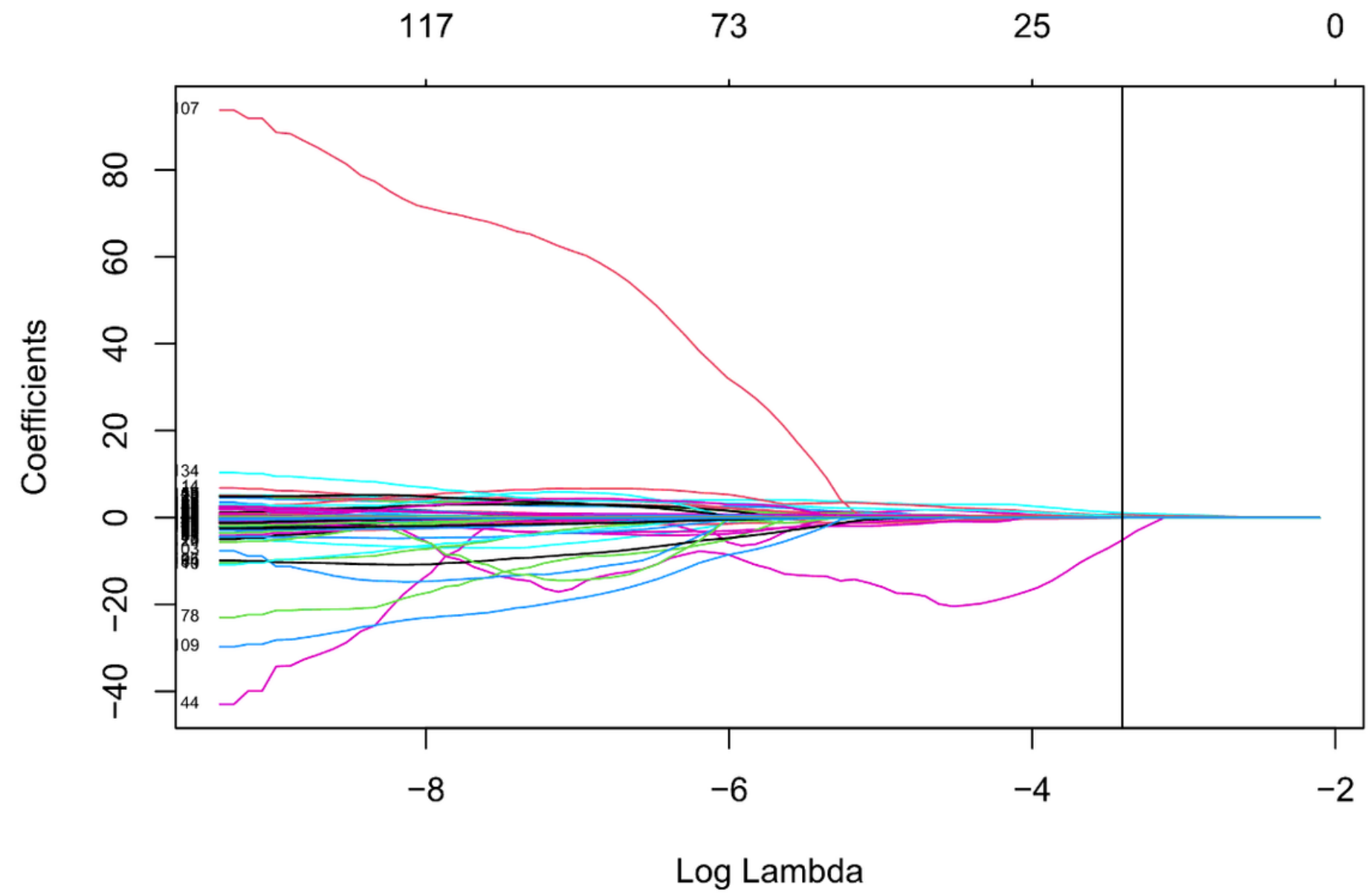

\section{Figure 3}

The Lasso coefficients of different features vary with the superparameter ( $\lambda$ value). The number above represents the number of features selected, and the black vertical line represents the 11 features with nonzero coefficients obtained. 


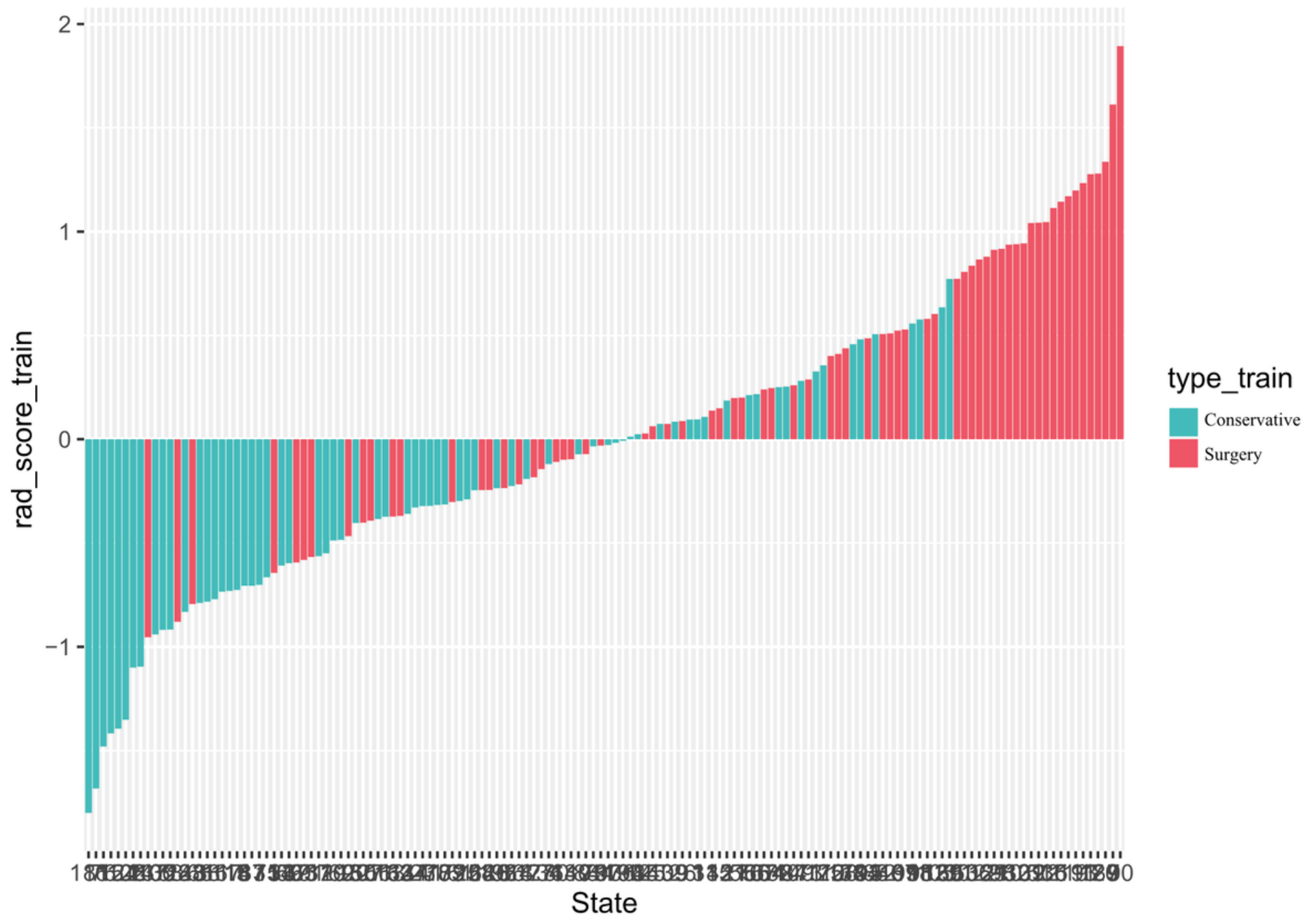

Figure 4

A radiomics label score for predicting treatment in the training group. 


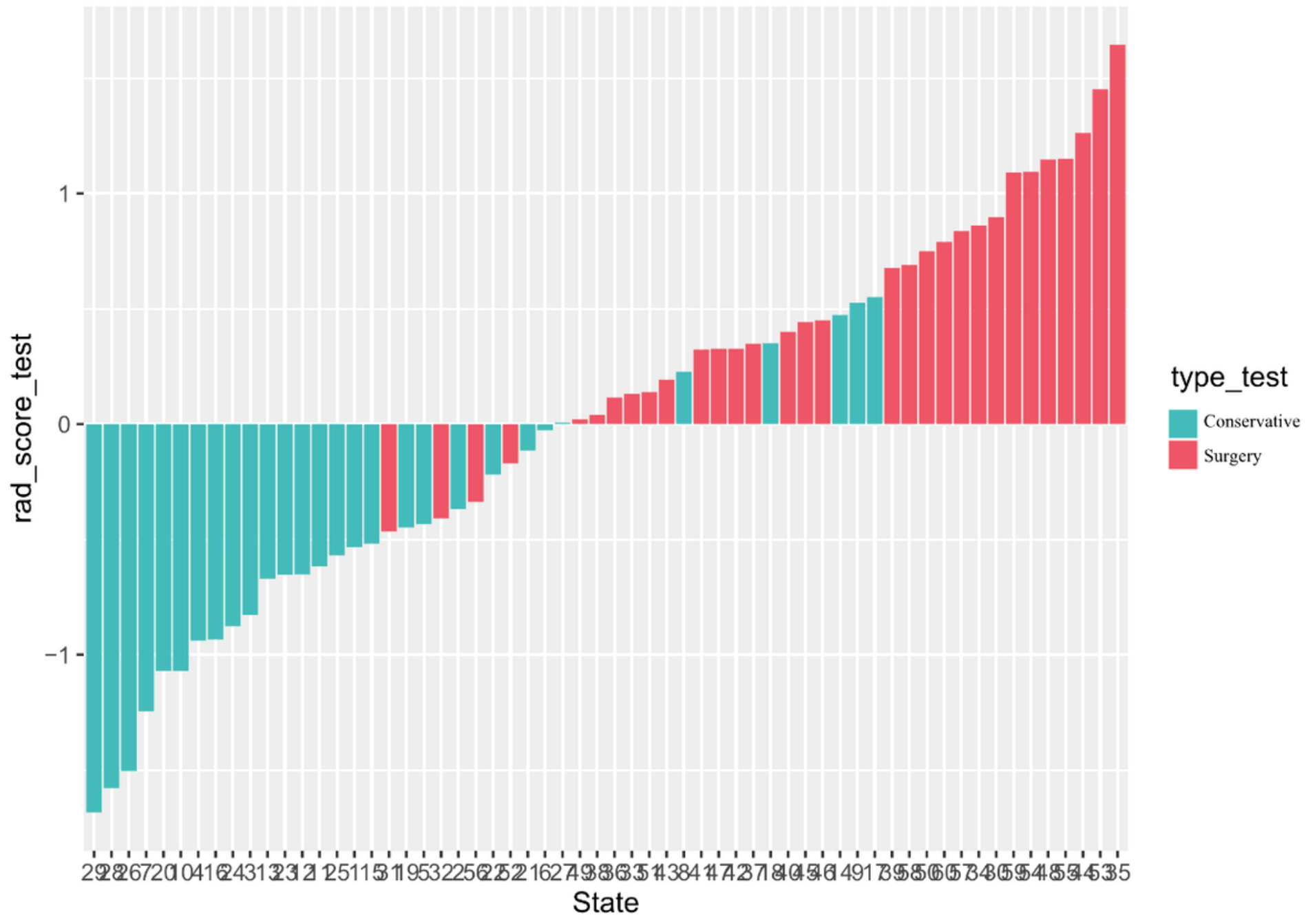

Figure 5

Verify the radiomics label score of the predicted treatment plan in the group. As can be seen from Fig. 4 and Fig. 5, the higher the score is, the more inclined the patients are to surgical treatment. A score greater than 0 represents surgical treatment, while a score less than 0 represents conservative treatment. 


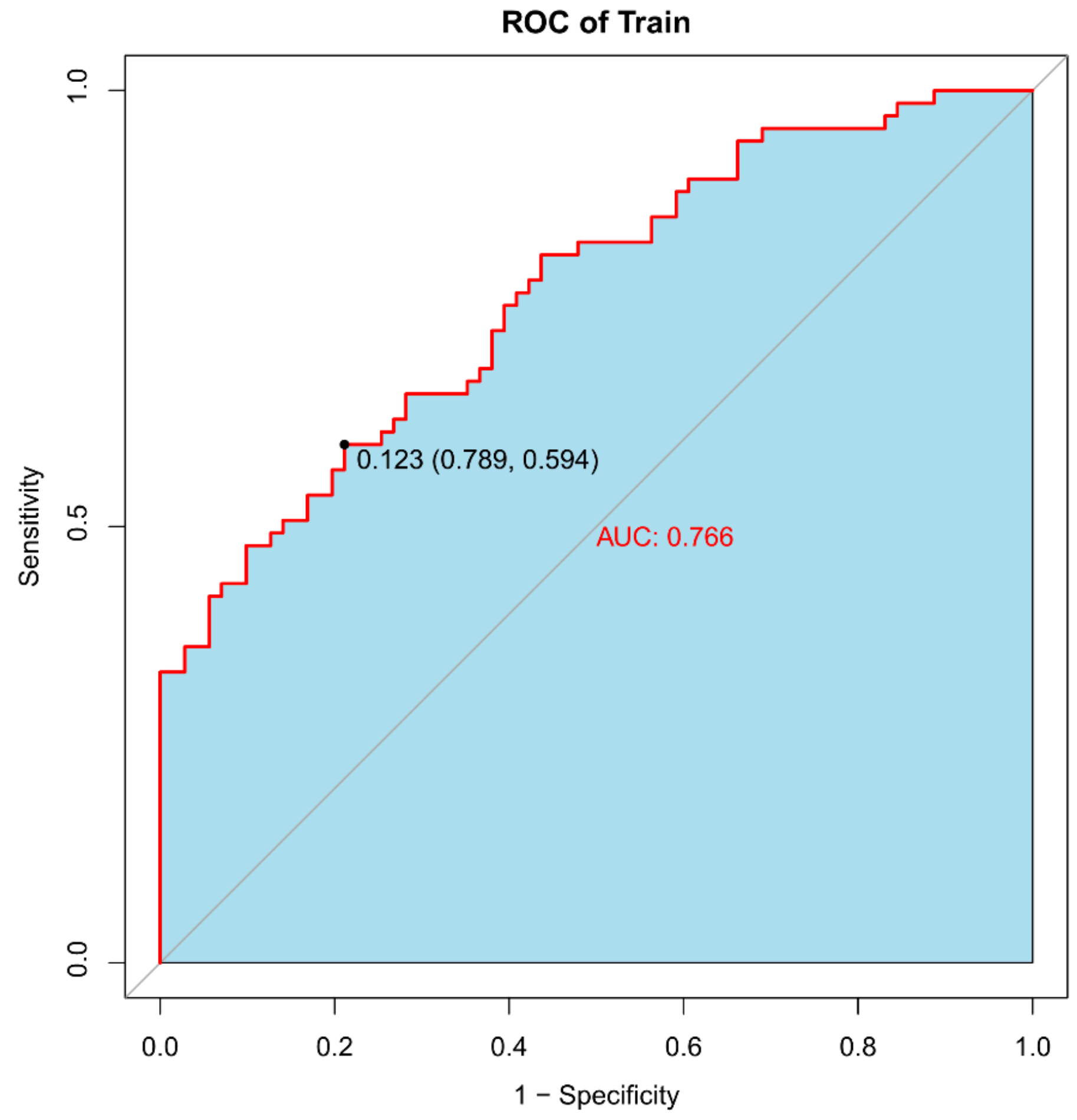

Figure 6

Radiomics label predicts ROC for treatment of lumbar disc herniation in the training group. 


\section{ROC of Test}

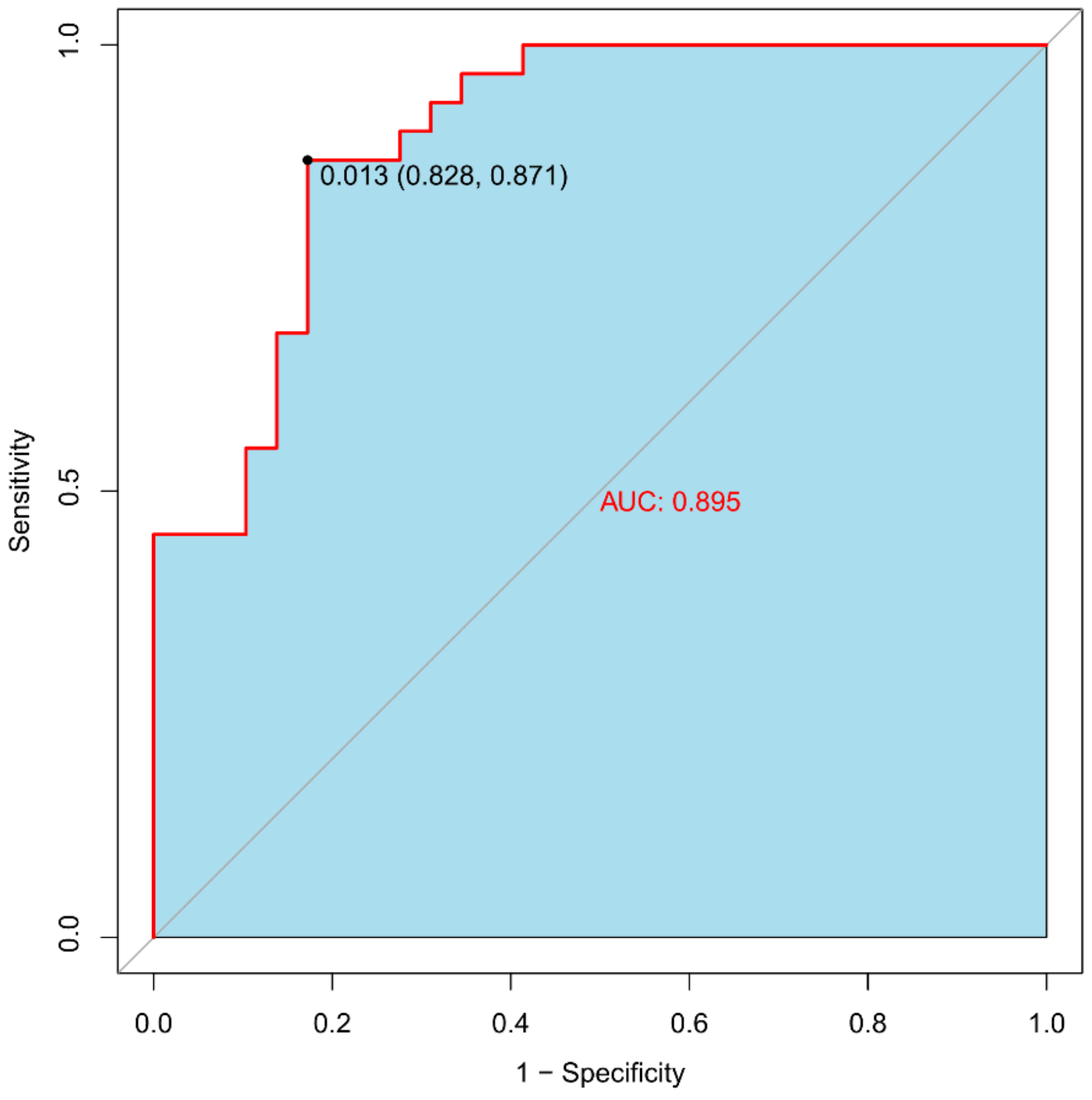

Figure 7

The ROC curve of the radiomics label predicting the treatment of lumbar disc herniation in the validation group was verified. 

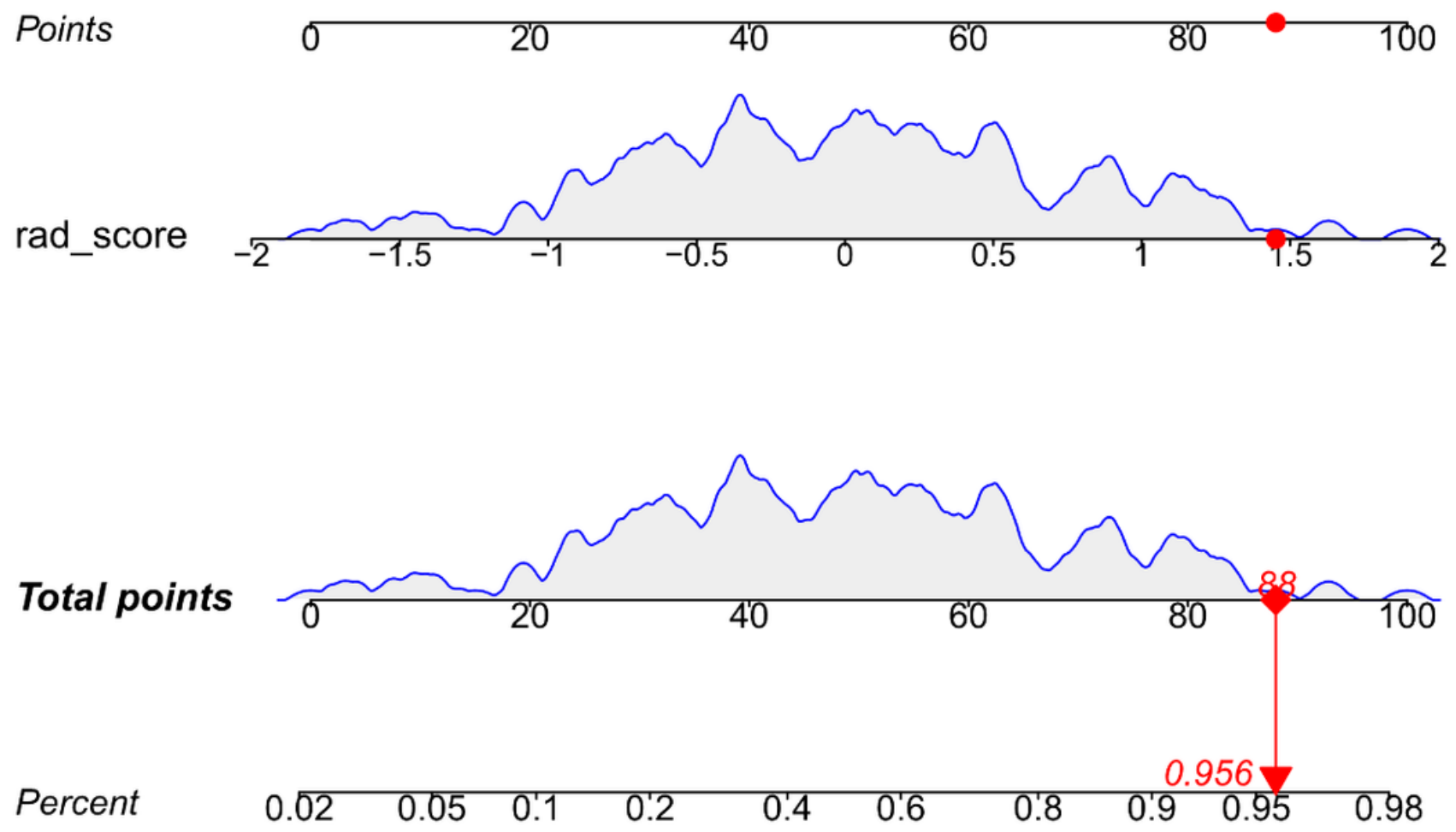

\section{Figure 8}

Nomogram of radiomics. The blue line is the distribution trend of radiomics labels and the red dot is the prediction example of a case. 


\section{ROC of Nomogram}

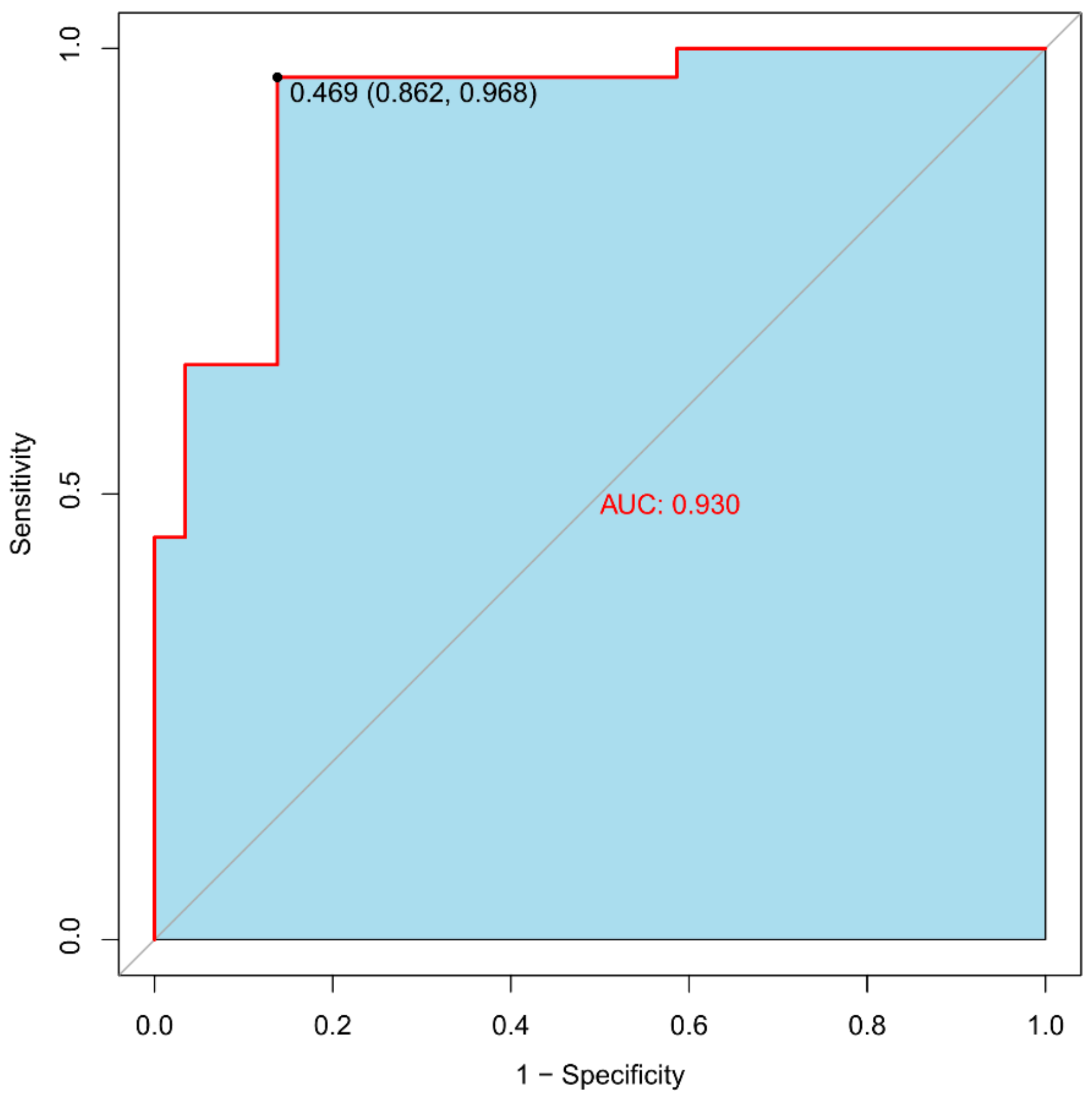

Figure 9

The ROC curve for predicting the treatment of lumbar disc herniation by radiomics nomogram. 


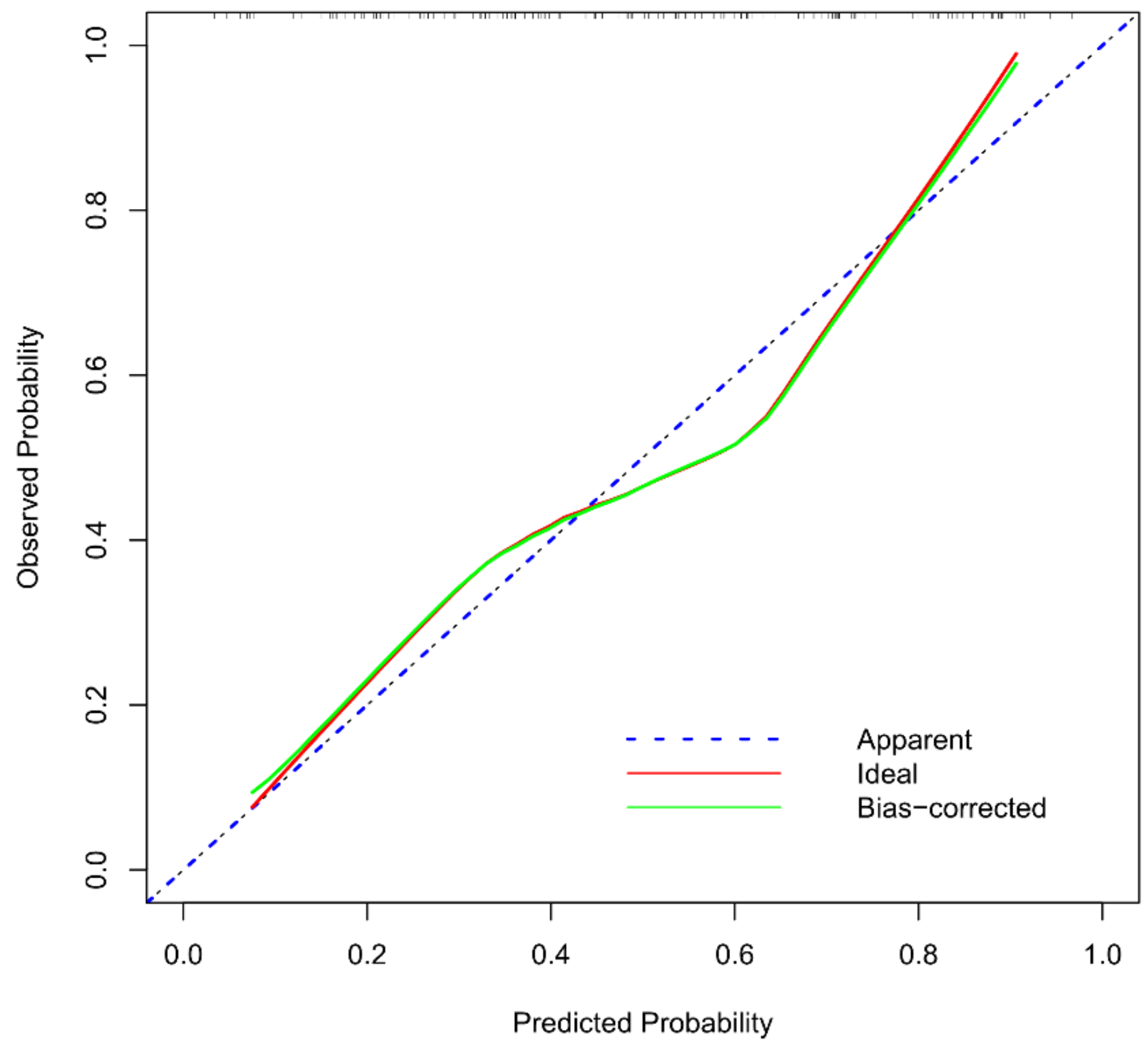

Figure 10

Calibration curve of radiomics nomogram in training group. 


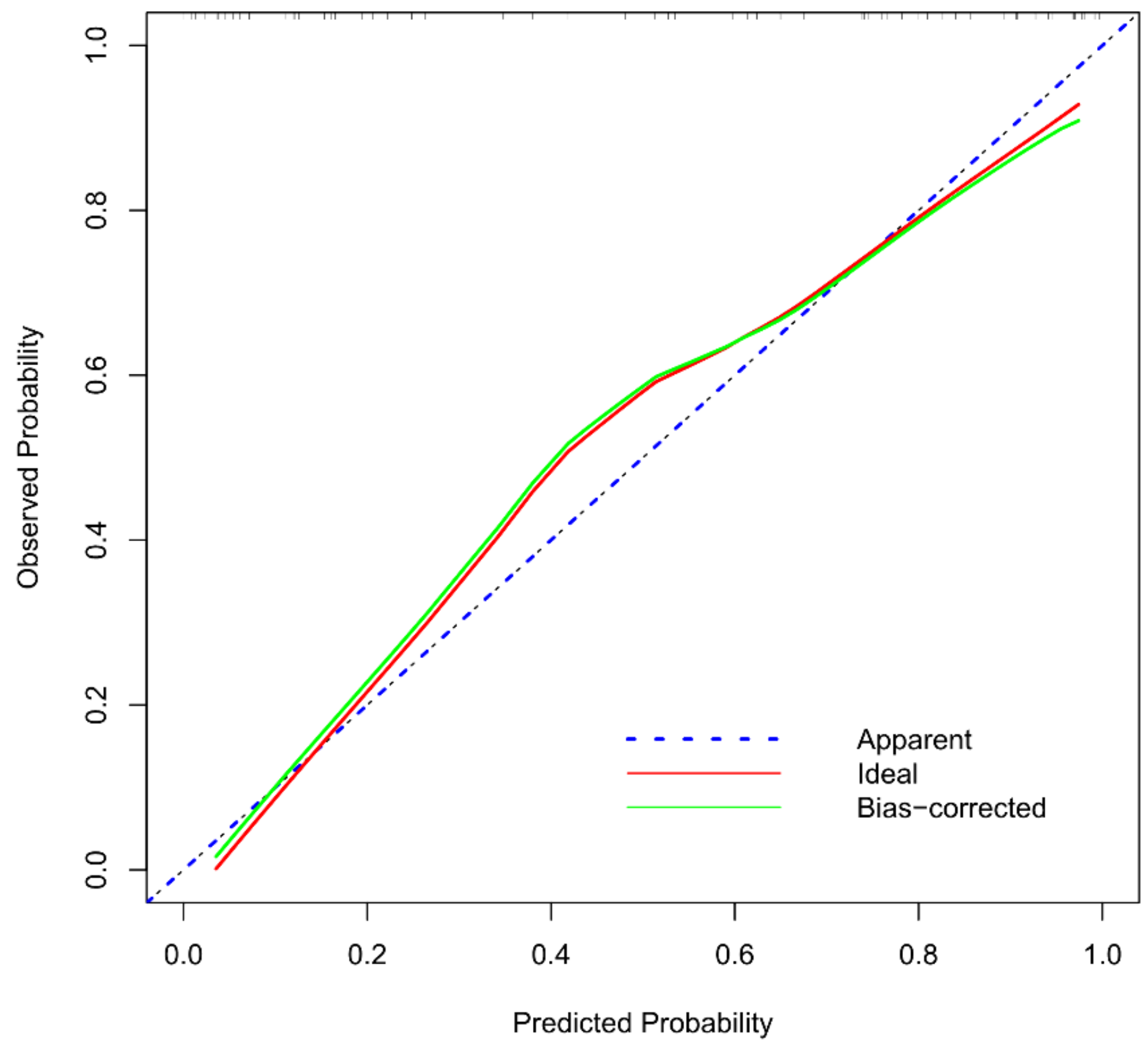

Figure 11

Verify the calibration curve of the nomogram of the radiomics. 


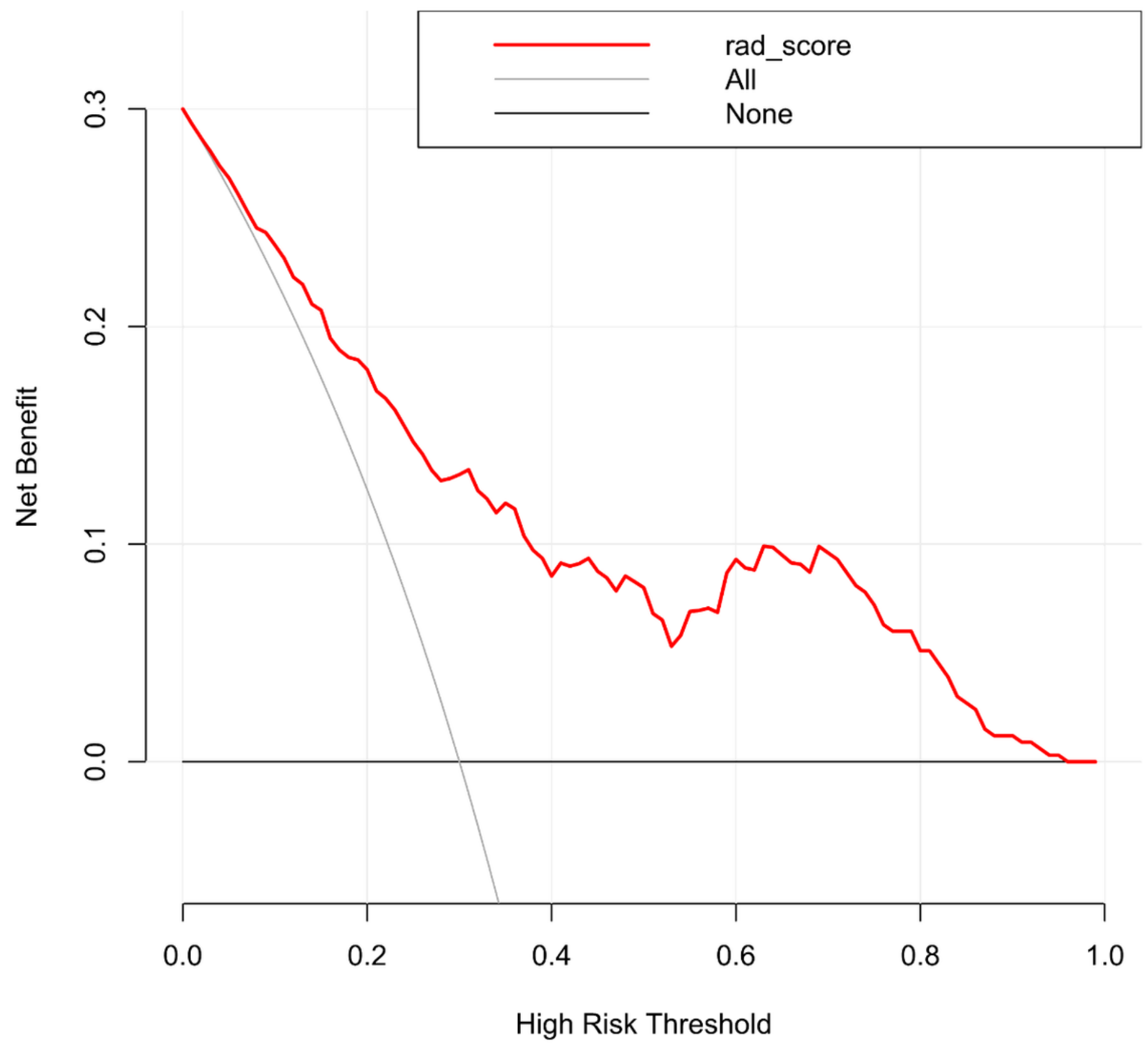

Figure 12

Decision-making curves of the predictive model in all patients with radiomics label nomogram. The decision curve represents the net benefit under different risk thresholds. 